ARTICLE

DOI: $10.1038 / \mathrm{s} 41467-018-04224-6$

OPEN

\title{
Air separation with graphene mediated by nanowindow-rim concerted motion
}

\author{
Fernando Vallejos-Burgos (10) ${ }^{1}$, François-Xavier Coudert (i) ${ }^{2} \&$ Katsumi Kaneko ${ }^{1}$
}

Nanoscale windows in graphene (nanowindows) have the ability to switch between open and closed states, allowing them to become selective, fast, and energy-efficient membranes for molecular separations. These special pores, or nanowindows, are not electrically neutral due to passivation of the carbon edges under ambient conditions, becoming flexible atomic frameworks with functional groups along their rims. Through computer simulations of oxygen, nitrogen, and argon permeation, here we reveal the remarkable nanowindow behavior at the atomic scale: flexible nanowindows have a thousand times higher permeability than conventional membranes and at least twice their selectivity for oxygen/nitrogen separation. Also, weakly interacting functional groups open or close the nanowindow with their thermal vibrations to selectively control permeation. This selective fast permeation of oxygen, nitrogen, and argon in very restricted nanowindows suggests alternatives for future air separation membranes.

\footnotetext{
${ }^{1}$ Center for Energy and Environmental Science, Shinshu University, Nagano 380-8553, Japan. ${ }^{2}$ Chimie ParisTech, PSL University, CNRS, Institut de Recherche de Chimie Paris, Paris 75005, France. Correspondence and requests for materials should be addressed to F.V.-B. (email: fvb@vallejos.cl)

or to K.K. (email: kkaneko@shinshu-u.ac.jp)
} 
S eparation processes comprise $40-70 \%$ of both capital and operating costs in modern industries ${ }^{1}$; increasing their efficiency then becomes critical. Separations based on distillation account for almost $8 \%$ of the total US energy consumption $^{2}$, thus having a very large carbon footprint due to carbon dioxide $\left(\mathrm{CO}_{2}\right)$ production ${ }^{3,4}$. The path to a sustainable future requires not only to improve the efficiency of the distillation processes but also to develop alternative separation technologies. Membrane-based techniques offer many benefits over conventional technologies based on phase transitions ${ }^{5}$. These benefits are a smaller equipment footprint, reduced mechanical complexity, and the requirement of $90 \%$ less energy than distillation ${ }^{2}$, resulting in much lower $\mathrm{CO}_{2}$ emissions. After mastering membrane technologies, separations based on phase changes will become obsolete.

Graphene is one of the recent superstars in the materials field and keeps growing in importance. With its synthesis processes continuously improving, we expect to achieve large-scale production with an exceptionally low concentration of defects. Due to its one-atom thickness, robustness, chemical stability, and ability to be converted into a sieve, one of its most important applications will be its usage as a membrane $e^{6-9}$.

Distillation is widely employed in the air separation industry to obtain high purity $\mathrm{N}_{2}, \mathrm{O}_{2}$, and noble gases, with significant amounts of energy spent on phase transitions ${ }^{10}$. Efficient membrane-based technologies have the potential to decrease the air separation industry's energy demand and reach higher product selectivities.

As pristine graphene sheets are impermeable even to the smallest gases such as helium ${ }^{11}$, it is thus necessary to introduce nanoscale windows (nanowindows) to transport and separate molecules. These nanowindows surpass conventional membranes, as their single-atom-thick wall provides almost negligible pore transport resistance, exhibiting ultra-fast molecular permeation ${ }^{12}$. We prefer the term nanowindows ${ }^{13,14}$ instead of nanopores, as the latter is a nanospace of deep-potential ready-toadsorb molecules, whereas nanowindows are atomically thick hole defects in a single-layer structure with the ability to open and close. Many methods can create nanowindows in graphene, e.g., ion bombardment ${ }^{15}$, template-synthesized mesh ${ }^{16}$, and simple high-temperature oxidation ${ }^{17}$.

The graphene nanowindow and especially its rim chemistry, featuring functionalized $\mathrm{C}$ edges, become critical factors determining the permeation rate and the selectivity of graphene membranes. The related computational studies in the literature, addressing the calculation of selectivity and permeation rates through nanowindows, are just as realistic as the membrane models employed. Extreme cases of an idealized nanowindow in graphene ${ }^{18-23}$ consist of removing some predetermined C atoms and modeling the remaining framework only through dispersion interactions. Although this can estimate the order of magnitude of permeation energies, it fails to estimate permeation rates due to their exponential relationship to energy. Moreover, most existing studies disregard the dynamic nature of the nanowindow and its rim chemistry, thus failing to predict the unprecedented permeation mechanism of graphene membranes.

Nanowindows are dynamic, as their rims vibrate and relax: the relaxation of a nanowindow in an annular polyaromatic molecule decreased two to five times the permeation energy barrier, depending on the permeating molecule ${ }^{24}$. Reducing the graphene framework to a non-periodic polyaromatic molecule also overemphasizes the role of relaxation, as the whole periodic
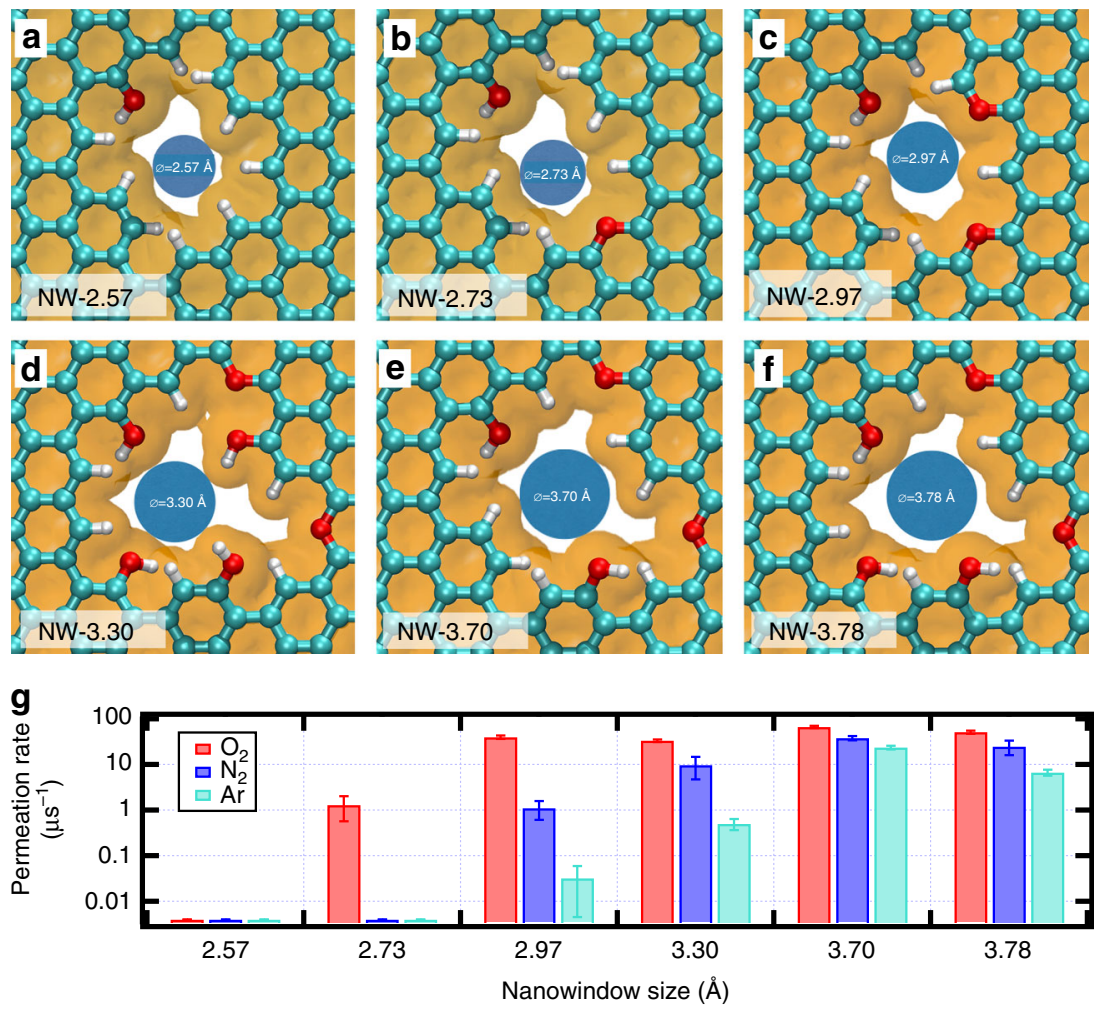

Fig. 1 Some quantum chemically allowed models of nanowindows. Each nanowindow is denoted as NW- $d$ where $d$ is van der Waals diameter of the opening in $\AA$, represented by brown shadings. Cyan, red and white atoms are $\mathrm{C}, \mathrm{O}$, and $\mathrm{H}$, respectively. Resulting sizes in $\AA$ were a 2.57; b 2.73; c 2.97; d 3.30; e 3.70; and $\mathbf{f} 3.78 . \mathbf{g}$ Permeation rate constants evaluated fitting the average of many MD runs in a canonical ensemble to a first order model. Temperatures are 90,77 , and $87 \mathrm{~K}$ for $\mathrm{O}_{2}, \mathrm{~N}_{2}$, and $\mathrm{Ar}$, respectively. Permeation rates barely shown indicate that no permeation events were observed during our simulations. Error bars represent uncertainty $\pm 1 \mathrm{SD}$. There is highly selective $\mathrm{O}_{2}$ permeation through a nanowindow smaller than the $\mathrm{O}_{2}$ 
framework is stiffer than the isolated molecule and gives rise to phonon vibrations. In the highly active metal organic framework (MOF) area, it has been demonstrated that the flexibility induced by adsorbed molecules also changes the structure of an $\mathrm{MOF}^{25,26}$. Incorporating flexibility into the models becomes crucial, as it is concurrent to molecular permeation.

A correct description of the rim chemistry of graphene nanowindows is then essential for practical atomistic simulations aiming to design highly selective membranes, because, as we will show, the presence of heteroatoms and defects induce an electric field around the nanowindow rim, which interacts with some permeating species. This important factor, known to have a big role in adsorption, e.g., in the case of zeolites ${ }^{27}$, is unaccounted for in idealized nanowindows. Moreover, functional groups such as hydroxyl, carboxyl, and carbonyl possess several orientations due to libration or torsion with respect to the nanowindow. Their dynamic orientations alter the nanowindow shape ${ }^{28}$ and effective size, which affects the permeation mechanism and its selectivity. Consideration of these functional groups has been limited mainly to stacked graphene oxides ${ }^{7}$.

Describing permeation only as dictated by geometric factors, such as the nanowindow size, becomes inaccurate if we consider that, owing to a thermal distribution of kinetic energies, molecules permeate even through a nanowindow smaller than its effective size. The geometrical size and shape of the nanowindow and the permeating molecule, and the chemistry and motion of the nanowindow rim taken altogether govern molecular permeation. In particular, the role of a concerted motion of nanowindow rims and their functional groups must be elucidated, because the dynamic motion of the nanowindow rim and the partial charge distribution produced by the heteroatoms give rise to molecular-recognition-type penetration, which can be applied to highly efficient molecular separation.

Taking on the challenge of separating molecules with similar sizes, but different interactions, gives information about their interactions with nanowindow rims. We choose the main air components, as their separation is industrially relevant and they exemplify different types of molecular interactions (dispersive and electrostatic), to discuss the impact of the nanowindow rims's assistance ${ }^{29,30}$. Our computer simulations show that realistic nanowindows can achieve four times the selectivity of experimentally reported membranes at a thousand times higher rate.

\section{Results}

Effective sizes and polar states of air molecules. In confined nanospaces, defining molecular dimension depending on the orientation that the molecule can adopt inside the restricted space is most appropriate. To permeate through a narrow nanowindow (see a nanowindow definition in Supplementary Fig. 1), two of the three orientation-dependent sizes will be constrained, and then the largest constrained dimension or second minimum dimension (MIN-2) in this case determines an effective molecular size for each gas molecule, which are 2.99, 3.05, and $3.63 \AA$ for $\mathrm{O}_{2}$, $\mathrm{N}_{2}$, and Ar, respectively ${ }^{31}$. A practical nanowindow sieve for air separation should range near these dimensions.

$\mathrm{N}_{2}$ and, to a lesser extent, $\mathrm{O}_{2}$ possess quadrupole moments that interact with other charges, such as the functional groups in the nanowindow rim. In contrast, Ar lacks electrostatic interactions. These differences create opportunities for a separation based on selective interactions.

Permeation rate through realistic nanowindows. With respect to molecular dynamics (MD) simulations, literature contains various cases of simplified nanowindows, e.g., without any heteroatom $^{18,21,23}$, a rigid framework ${ }^{20-22}$, or too idealized rim symmetries ${ }^{18,19}$. To improve the phenomenological description, we created more realistic and feasible nanowindows by including functional groups and a totally flexible graphene framework.

Six different nanowindow models were designed (see Fig. 1a-f) with a size similar to the permeating molecules. Notation for each nanowindow is NW- $d$, where $d$ is the aperture size ranging from 2.57 to $3.78 \AA$. We followed three considerations in building the nanowindows: first, our nanowindows are created by oxidation in air and thus the C-edges are passivated with $\mathrm{H}$ or $\mathrm{O}$ atoms. Passivation of the free C-edges results in a $\mathrm{MJ} \mathrm{mol}^{-1}$ order of decrease in system energy (see Supplementary Table 1). Second, the oxygen content increases with oxidation ${ }^{21,32}$ with the heteroatoms existing mainly as ubiquitous hydrogen ${ }^{33}(\mathrm{R}-\mathrm{H})$, phenol $^{34-37}(\mathrm{R}-\mathrm{OH})$, and ether ${ }^{37-39}\left(\mathrm{R}-\mathrm{O}-\mathrm{R}^{\prime}\right)$; and third, the aperture sizes are between 2.57 and $3.78 \AA$, which reasonably encloses the range of molecular sizes of interest in air separation.

Permeation rates were evaluated for each nanowindow from MD simulations in the canonical ensemble. The simulation cell consists of two compartments separated by graphene with a nanowindow, where initially one compartment is filled with gas. Rates reported are the rate constants considering first-order permeation kinetics (see derivation in Supplementary Note 1 and Supplementary Fig. 2). The permeation steps and mechanisms
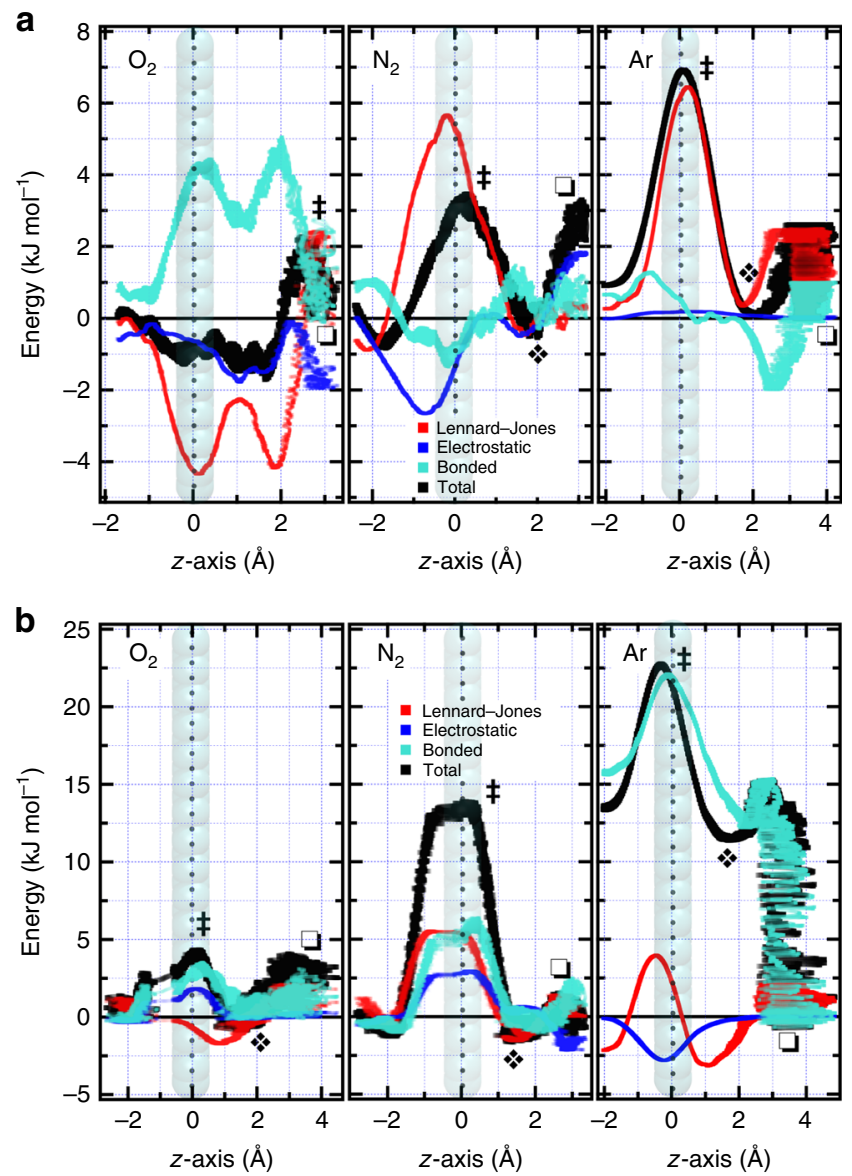

Fig. 2 Enthalpy contributions to permeation through nanowindows. a, b Permeation energy barriers of $\mathrm{O}_{2}(90 \mathrm{~K}), \mathrm{N}_{2}(77 \mathrm{~K})$, and $\mathrm{Ar}(87 \mathrm{~K})$ through NW-3.30 a and NW-2.97 b. The $z$-axis is perpendicular to the graphene layer, located at $z=0$. Total energy (black line) sums the contributions from Lennard-Jones (red), electrostatic (blue), and bonded interactions (turquoise). Bonded interaction energy considers bonds, angles, dihedrals, and impropers. Square, diamond, and double dagger symbols represent adsorption on the basal plane, adsorption on top of the nanowindow, and a high-energy state across the graphene plane, respectively 
resulting in a nanowindow crossing were already analyzed in several excellent papers ${ }^{19,40-42}$. Briefly, for a permeation event to occur, the molecule first needs to be transported from the gas phase to the adsorbed state near a nanowindow in the graphene basal plane. Then, it needs to leave the basal plane and move to the top of the nanowindow, in an incipient permeation position. Finally, it propagates through the nanowindow (see all steps in Supplementary Fig. 3) and desorbs on the other side of the membrane. In general, the gas permeation rate constants (see Fig. 1g) decrease with nanowindow size. Due to their large size, NW-3.78 and NW-3.70 have a high permeation rate and low separation selectivity. Narrowing the size to $3.30 \AA$ decreases the Ar permeation rate constant by a factor of 50 and increases the $\mathrm{N}_{2} /$ Ar selectivity to 20 , evidencing a molecular sieving regime.

Deeper observation of the tendency in the permeation rate constants evidences some (surprising) facts. For example, $\mathrm{O}_{2}$ permeates faster through NW-2.97 than NW-3.30 and $\mathrm{N}_{2}$ permeates faster through NW-3.70 than NW-3.78. In both cases, this is due to the increased population of permeating molecules on top of the nanowindow (density profiles for the case of $\mathrm{O}_{2}$ are shown in Supplementary Fig. 4). $\mathrm{O}_{2}$ population on top of the nanowindow is five times larger in NW-2.97 than in NW-3.30; in the case of $\mathrm{N}_{2}$, it was 1.7 times larger. Finally, a faster $\mathrm{Ar}$ permeation rate of NW-3.70 compared with NW-3.78 occurred due to the lower system energy when $\operatorname{Ar}(\mathrm{MIN}-2$ size is $3.6 \AA$ ) is inside a non-sterically confined nanowindow: a calculation of energy profile showed intra-nanowindow maximum energies of an Ar atom of $-953 \mathrm{~K}$ and $-708 \mathrm{~K}$ for NW-3.70 and NW-3.78, respectively. This energy difference readily explains the different permeation rates.

Permeation rate and molecular size. Surprisingly, molecules permeate even if their effective size is larger than the nanowindow itself, as the high-energy (or transition) state occurs only temporarily and can be overcome by kinetic energy fluctuations of the incoming particle. a

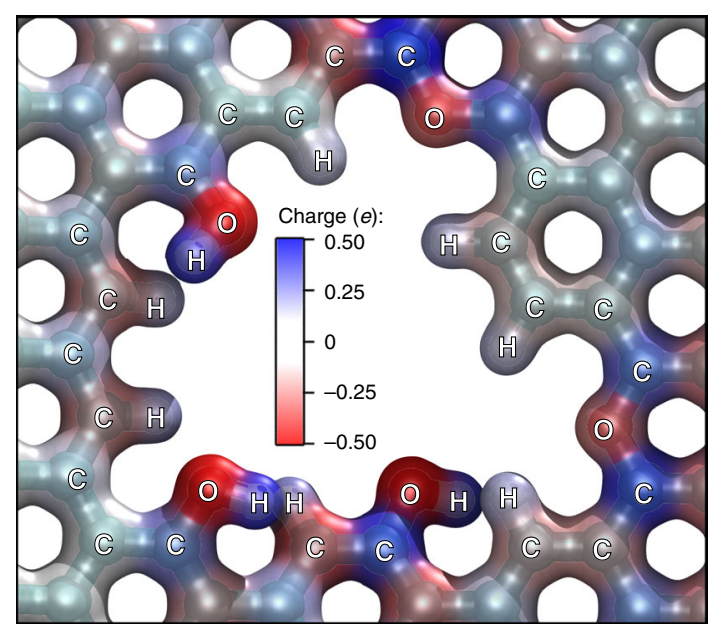

c

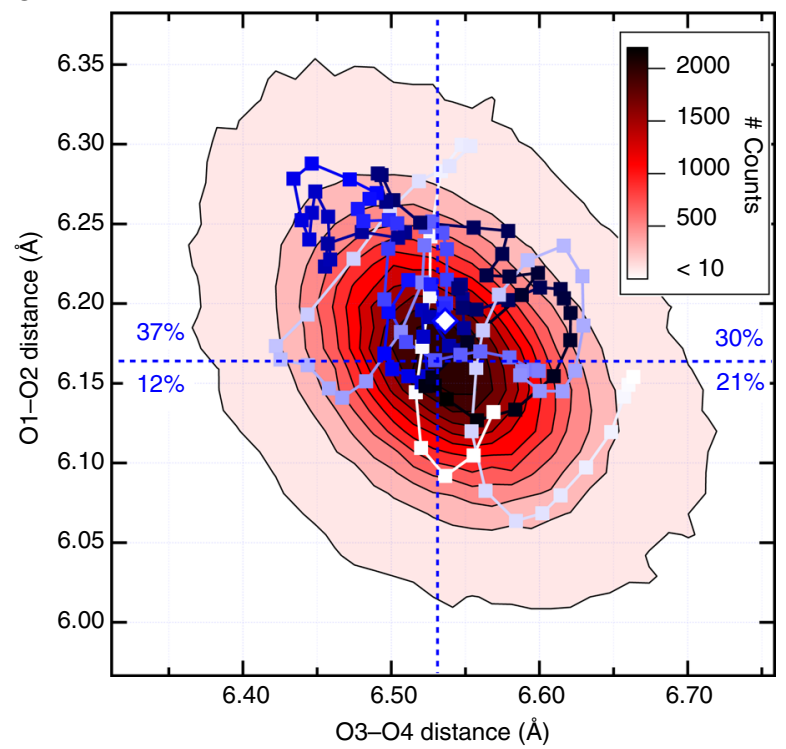

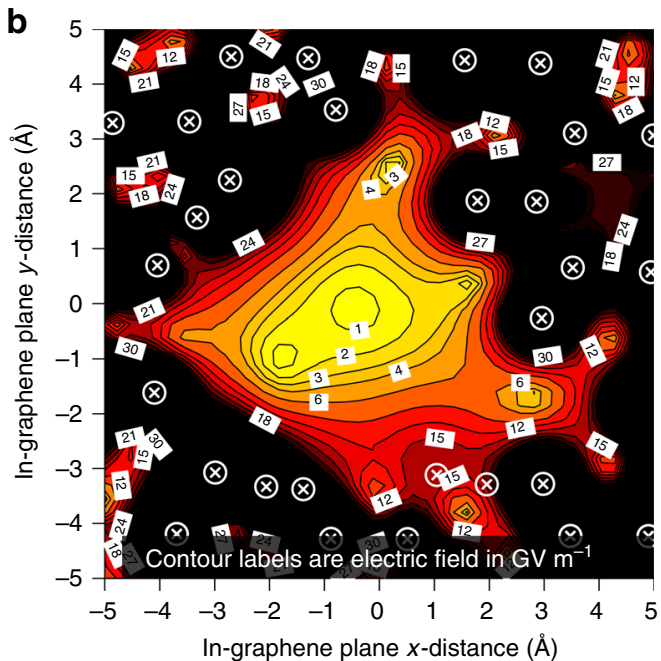

d

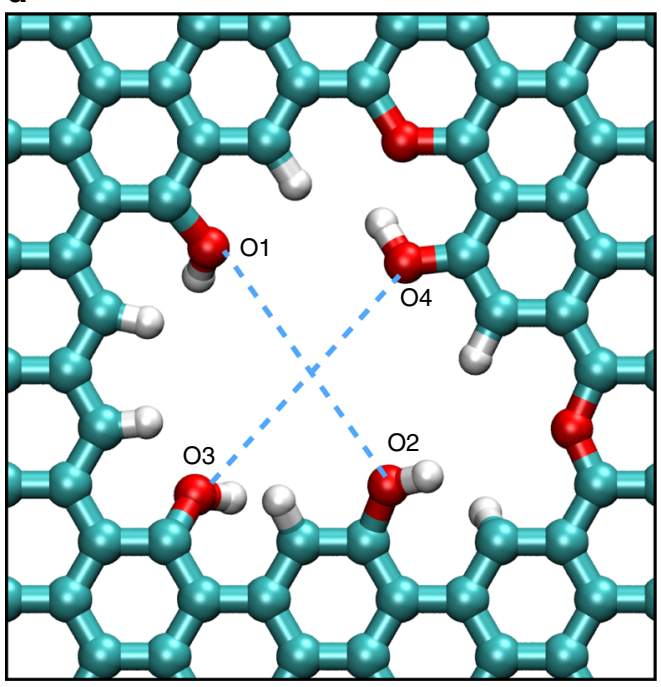

Fig. 3 Large electrostatic field and breathing of two nanowindows. a Partial charges in NW-3.78 nanowindow calculated according to MK scheme. b Contour plot for in-graphene plane magnitude of the electric field in $\mathrm{GV} \mathrm{m}{ }^{-1}$ calculated from partial charges shown in a for NW-3.78. Circles with crosses indicate position of atoms. c 2D histogram contour of the distances between two pairs of $\mathrm{O}$ atoms on opposite sides of the rim during an MD simulation in NW-3.30. Vibration is asymmetric with a Pearson correlation coefficient between $01-02$ and $03-04$ of - 0.38. The blue-gradient path corresponds to the distances when a $\mathrm{N}_{2}$ molecule permeated through the nanowindow. Darker marks correspond to $\mathrm{N}_{2}$-center of mass closer to the nanowindow center in the z-direction. d Atomic numbering used to calculate lengths in c. Cyan, red and white atoms are $\mathrm{C}, \mathrm{O}$, and $\mathrm{H}$, respectively 
This is evident in the case of nanowindows NW-2.97 and NW3.30 where even Ar permeates, at a size (MIN-2 size is $3.63 \AA$ ) at least $10 \%-22 \%$ larger than the nanowindow. In these cases, the permeation transition state lasts less than a picosecond in our simulations at $87 \mathrm{~K}$. This phenomenon is reminiscent of MOF: ZIF-8 with its extraordinary framework flexibility, capable of adsorbing hydrocarbons larger than its nominal aperture size $e^{43}$.

A reaction pathway for a small gas molecule being pulled through a nanowindow (see Fig. 2) has generally two local energy minima and a single transition state. Local minima occur when the molecule is adsorbed on the basal plane at $z$ equals $3.3 \AA$ or on top of the nanowindow at around $2 \AA$. The high-energy state occurs in the in-graphene plane at $z$-axis is $0 \AA$ when the molecule squeezes through the nanowindow rim. $\mathrm{O}_{2}$ freely permeates through nanowindow NW-3.30 due to its smaller size, lacking an in-graphene plane transition state (Fig. 2a). The enthalpy barriers for crossing nanowindows of similar size as the permeating molecule are in the order of $3-6 \mathrm{~kJ} \mathrm{~mol}^{-1}$. For Ar, the main contributor to the total enthalpy at the transition state is dispersion interaction due to its repulsion term, whereas for $\mathrm{N}_{2}$ this repulsion is counterbalanced by a strong electrostatic stabilization.

When permeating a narrower nanowindow (see Fig. 2b), $\mathrm{O}_{2}$ and $\mathrm{N}_{2}$ face one energetic barrier of 4 and $13 \mathrm{~kJ} \mathrm{~mol}^{-1}$, respectively. However, Ar exhibits super-slow permeation due to two consecutive energy barriers that deform the functional groups when displaced from the basal plane to the top of the nanowindow, and then another deformation by crossing the graphene layer.

Rim heteroatoms induce a strong electrostatic field. The different electronegativities of the $\mathrm{H}$ or $\mathrm{O}$ atoms bonded to $\mathrm{C}$ at the rim of the nanowindow, together with the addition of defects in the graphene network, induce heterogeneity in the electronic density of the nanowindow rim atoms. These partial charges (see Fig. 3a) along the rim create an electrostatic field around the nanowindow of $\mathrm{GV} \mathrm{m}^{-1}$ magnitude (Fig. 3b). This interacts differently with the quadrupole moments of $\mathrm{O}_{2}$ and $\mathrm{N}_{2}$.

The effect of this large electric field is evident by comparing electrostatic potential energies of air molecules while permeating the nanowindow (see Fig. 2a), as $\mathrm{O}_{2}, \mathrm{~N}_{2}$, and Ar interactions are weak, strong, and non-existent, respectively. In the case of $\mathrm{N}_{2}$, the dispersion repulsion, $5.6 \mathrm{~kJ} \mathrm{~mol}^{-1}$, is strongly counteracted by an electrostatic stabilization of $-2.7 \mathrm{~kJ} \mathrm{~mol}^{-1}$, resulting in a total energy requirement of only $3.1 \mathrm{~kJ} \mathrm{~mol}^{-1}$. However, in the case of Ar, the main contribution to total energy arises from its repulsion with nanowindow rim atoms.

Nanowindows breathe and relax. Graphene has phonon motion and local oscillations, which give rise to concerted vibrations in the nanowindow rim (see Supplementary Movie 1). These vibrations alter the effective size and shape of a nanowindow, thus determining its permeation properties. The distribution of these concerted vibrations at the rim can be visualized via a twodimensional contour histogram of the distances between opposing $\mathrm{O}$ atoms during a simulation (see Fig. $3 \mathrm{c}-\mathrm{d}$ ). Although a high concentration of distances is at the center of the plot, thermal energy creates fluctuations of these atomic distances in about 0.1 $\AA$. The Lennard-Jones (LJ) energy difference when an Ar atom approaches four O atoms from $3.30 \AA$ to $3.25 \AA$ shows that this concerted motion indeed involves a considerable energy penalty of $0.5 \mathrm{~kJ} \mathrm{~mol}^{-1}$.

The $\mathrm{O}-\mathrm{O}$ pair distances were followed while $\mathrm{N}_{2}$ permeated through the nanowindow (path in Fig. 3c). As bond vibrations are around 10 times faster than permeation, there is a large fluctuation of interatomic distances. Most permeation occurred when the shorter $\mathrm{O}-\mathrm{O}$ distance was above its average, due to the intuitive fact that the nanowindow vibration is sterically constrained when a molecule is inside. The gas permeation then becomes concerted to the nanowindow vibration, allowing the rim to enlarge due to a molecular crossing event. This is clear by comparing against a rigid nanowindow (see in Table 1 the rigid nanowindows labelled $\mathrm{NW}-n \mathrm{C}$, with $n$ the number of $\mathrm{C}$ atoms removed) where frameworks with similar selectivity have much lower permeation rates. Such coherent vibration is very similar to that observed in the framework dynamics of small-pore zeolites, where it is called a "window breathing" mode, which significantly impacts guest diffusion and molecular sieving ${ }^{44}$. This asymmetrical nanowindow breathing mode is employed in the separation of molecules by shape.

Rotations of functional groups open or close nanowindows. $\mathrm{He}$ et al. ${ }^{28}$ demonstrated that nanowindows with negatively charged carboxylate groups present asymmetric permeation energy profiles at each side of the graphene wall. This was due to different

Table 1 Nanowindows separate air much faster than conventional membranes

\begin{tabular}{|c|c|c|c|c|}
\hline Membrane type & Rate (GPU) & Selectivity $\mathrm{O}_{2} / \mathrm{N}_{2}$ & Temperature (K) & Reference \\
\hline Graphene nanowindow NW-2.73 & 27,100 & 45 & 195 & This work \\
\hline Graphene nanowindow NW-2.97 & 278,000 & 7.3 & 195 & This work \\
\hline Graphene nanowindow NW-2.73 & 10,040 & 20 & 300 & This work \\
\hline Graphene nanowindow NW-2.97 & 60,500 & 5.0 & 300 & This work \\
\hline Graphene nanowindow NW-8C & 1950 & 62 & 300 & This work \\
\hline Graphene nanowindow NW-10C & 25,070 & 6.8 & 300 & This work \\
\hline Graphene nanowindow NW-13C & $1,467,800$ & 1.1 & 300 & This work \\
\hline Polysulfone hollow fiber & 43 & 5.2 & 323 & Kesting et al. ${ }^{47}$ \\
\hline Asymmetric polysulfone hollow fibers & 8.8 & 5.8 & 297 & Pesek and Koros ${ }^{48}$ \\
\hline 6FDA-durene hollow fiber & 64 & 3.20 & 298 & Chung et al. ${ }^{49}$ \\
\hline 6FDA-durene hollow fiber & 1512 & 1.09 & 298 & Chung et al. ${ }^{49}$ \\
\hline Matrimid 5218 polyimide hollow fiber & 7 & 7.5 & 297 & Clausi and Koros ${ }^{50}$ \\
\hline Matrimid 5218 polyimide hollow fiber & 33.7 & 6.0 & 297 & Clausi and Koros 50 \\
\hline Modified polyphenylene oxide film & 648 & 3.9 & 308 & Sterescu et al. ${ }^{51}$ \\
\hline Polyimide hollow fiber & 300 & 3 & 333 & Liu et al. $^{52}$ \\
\hline Polydimethylsiloxane hollow fiber & 60 & 1.8 & 298 & Prajapati et al. ${ }^{53}$ \\
\hline Polydimethylsiloxane hollow fiber & 7 & 7 & 298 & Prajapati et al. ${ }^{53}$ \\
\hline
\end{tabular}


ways that the carboxylate groups orient toward the graphene plane, creating different environments on each side of the graphene wall. Later, Drahushuk et al. ${ }^{41}$ experimentally found that nanowindow-rim bonds can be chemically rearranged, thus modifying the nanowindow shape and consequently the permeation barrier to achieve selective gas permeation. It is then required for computer simulations to sample all allowed configurations of the nanowindow by considering a flexible framework.

Similar to these out-of-plane configurations, other out-of-plane functional groups dynamically switch their orientation. We analyzed the effect of dihedral orientation of hydroxyl groups in the nanowindow rim (e.g., O1-O4 in Fig. 3d). A set of simulations run for $\mathrm{N}_{2}$ permeation through the flexible NW-3.30 framework (see Fig. 4a) show large-scale fluctuations, with a large spread in permeability rate constants $\left(k=10 \pm 5 \mu \mathrm{s}^{-1}\right)$. During all our results presented so far, the full system was allowed to vibrate. The $\mathrm{H}$ atoms (which only electrostatically interact with $\mathrm{N}_{2}$ and $\mathrm{O}_{2}$ ) in the hydroxyl groups are free to rotate through $\mathrm{H}-\mathrm{O}-\mathrm{C}-\mathrm{C}$ dihedrals, temporarily locking the $\mathrm{H}$ in local energy minima configurations. In contrast, starting the simulations as fully flexible and then quenching the nanowindow framework rigid evidences the importance of these configurations. It allowed identifying three distinct rate regimes of permeation separated by orders of magnitude. A fast permeation regime $\left(k=28 \pm 11 \mu \mathrm{s}^{-1}\right.$, Fig. $4 \mathrm{~b}$ ) occurs when the $\mathrm{OH}$ atom pairs point toward opposite sides of the graphene plane (Fig. 4e, with O-atoms notation in Fig. 3d), allowing a larger nanowindow space to be opened for the permeating $\mathrm{N}_{2}$. In all these cases, $\mathrm{H}-\mathrm{O} 2$ pointed toward the reader, creating a favorable environment for permeation. In the moderate rate permeation regime $\left(k=3.3 \pm 0.5 \mu \mathrm{s}^{-1}\right.$, Fig. $\left.4 \mathrm{c}\right)$, the $\mathrm{OH}$ atom pairs point in opposite out-of-graphene-plane sides and converging graphene-plane direction or same out-of-grapheneplane side but diverging graphene-plane directions (see Fig. 4f). Finally, in the slow rate permeation regime $\left(k=0.1 \pm 0.2 \mu \mathrm{s}^{-1}\right.$, Fig. $4 \mathrm{~d}$ ), both atom pairs choke the nanowindow by pointing and also converging in the same direction (see Fig. 4g). Changes in $\mathrm{O} 3-\mathrm{H}$ hydroxyl group orientation had no effect on permeation.

Then, through torsion of functional groups $\mathrm{H}-\mathrm{O} 1$ and $\mathrm{H}-\mathrm{O} 2$, the nanowindow electrostatically (as $\mathrm{H}$ is just modeled as a point charge) closes to $\mathrm{N}_{2}$. This shows that the $\mathrm{OH}$ group position closes the nanowindow to electrostatically interacting molecules, showing strong potential for enhanced separations by including other functional groups in the rim.

As $\mathrm{O}_{2}$ is smaller than $\mathrm{N}_{2}$, it is natural to require a smaller nanowindow to observe a strong effect of the rim functional groups movement on permeation. This sieving has to occur where the molecular crossing is constricted by the rim, i.e., NW-2.73 and smaller for $\mathrm{O}_{2}$ (notice the decrease in $\mathrm{O}_{2}$ permeation rates in Fig. 1). As in the previous case with $\mathrm{N}_{2}$, permeation with the graphene framework locked rigid in different energy minima, showing the importance of the functional group vibration in even narrower nanowindows. Two different $\mathrm{O}_{2}$ permeation cases occur in NW-2.73 depending on the mutual orientation of the opposing armchair hydrogen pairs in the nanowindow rim: (i) an open regime (see Fig. 5a, rate constant $1.8 \mu^{-1}$ ) when H-pairs flap away from the graphene layer in opposing directions, allowing
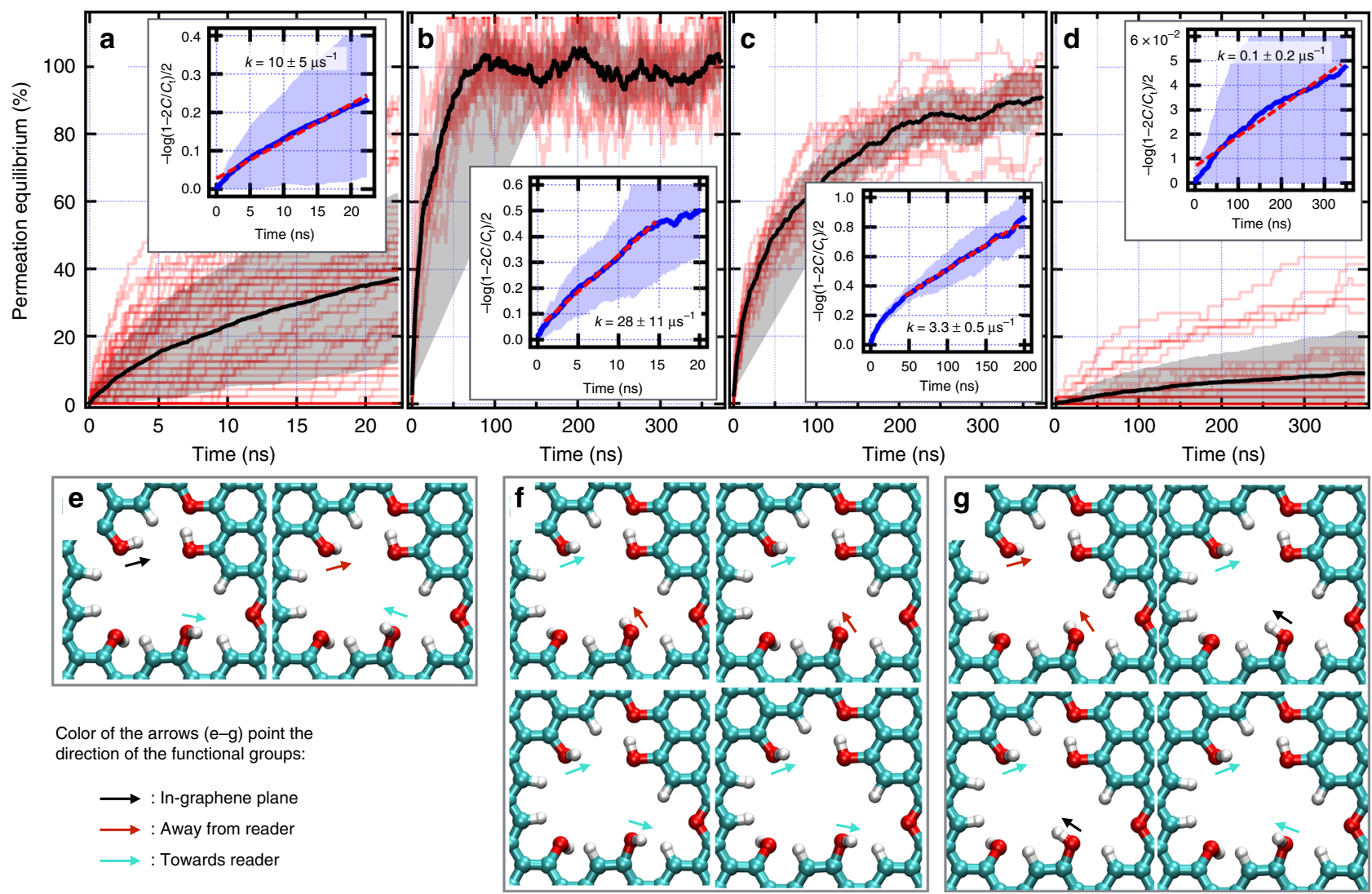

Fig. 4 Effect of nanowindow dihedral rotation on $\mathrm{N}_{2}$ permeation rates. a-d Molecular dynamics results of $\mathrm{N}_{2}$ permeability at $77 \mathrm{~K}$. Thin red lines are single runs, the thick black line is the average of all runs in red and the shaded area is standard deviation. The inset is the linearization of all data including a linear fit to the average. $k$ is the permeation rate constant where uncertainty is 1SD. Results are divided in a all results for a flexible nanowindow framework and for a rigid framework grouped in $\mathbf{b}$ fast, $\mathbf{c}$ medium, and $\mathbf{d}$ slow. $\mathbf{e - g}$ Show several representative orientations of functional groups for $\mathbf{b}$, $\mathbf{c}$, and $\mathbf{d}$ regimes, respectively. Colored arrows indicate the orientation of $\mathrm{O}(1-2)-\mathrm{H}$ bond. Cyan, red, and white atoms are $\mathrm{C}, \mathrm{O}$, and $\mathrm{H}$, respectively. All these results correspond to NW-3.30 

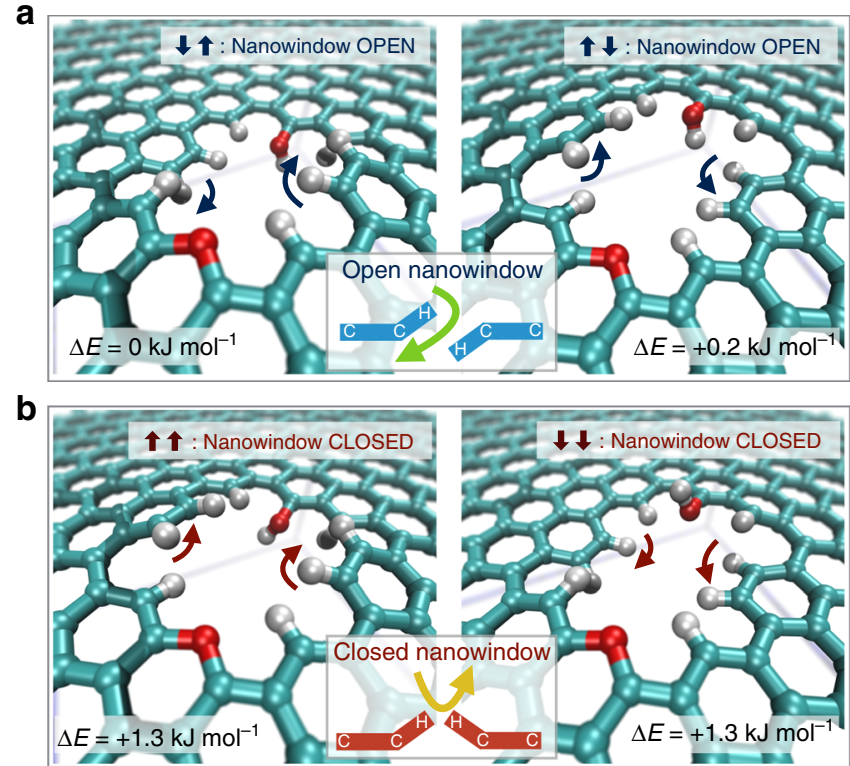

Fig. 5 Functional group flapping mechanism in a small nanowindow. The nanowindow can be in an open or closed states for allowing $\mathrm{O}_{2}$ permeation. a Open states. Pairs of hydrogen point in different directions. b Closed states. Pairs of hydrogen point in the same direction. Arrows indicate the directions where the $\mathrm{H}$-pairs point. Energy difference is respective to the structure a, left. Cyan, red, and white atoms are $\mathrm{C}, \mathrm{O}$, and $\mathrm{H}$, respectively. Insets illustrate the equivalence between flapping movement and the opening/closing of gates. These figures correspond to $\mathrm{O}_{2}$ permeation at $90 \mathrm{~K}$ through NW-2.73 permeation of $\mathrm{O}_{2}$ and (ii) an impermeable regime (see Fig. 5b, rate constant less than $0.001 \mu \mathrm{s}^{-1}$ ) when both $\mathrm{H}$-pairs flap toward the same direction and close the nanowindow. Open positions are slightly more favorable $\left(\Delta E=-1.3 \mathrm{~kJ} \mathrm{~mol}^{-1}\right)$, which is confirmed by their number of occurrences. This flapping movement is correctly sampled in the flexible framework, as its rate constant contains contributions from both closed and open states of the nanowindow.

Narrow nanowindows separate air ultra-fast and selectively. Selectivity was first evaluated with the ratio of single gas permeation. A very oxygen-selective and fast-permeating nanowindow is NW-2.97: its permeation rate constant is $47 \mu \mathrm{s}^{-1}$, which translates into $600 \mathrm{~m}^{3} \mathrm{STP} \mathrm{min}^{-1} \mathrm{~m}^{-2}$ and a selectivity larger than 50 for $\mathrm{O}_{2}: \mathrm{N}_{2}$ separation (and 1500 for $\mathrm{O}_{2}$ :Ar). Narrower membranes, such as NW-2.73, allow slow permeation of $\mathrm{O}_{2}$, while totally blocking $\mathrm{N}_{2}$ and $\mathrm{Ar}$ in the $200 \mathrm{~ns}$ timescale of our simulations.

In following more realistic experiments, a model mixture of air $\left(\mathrm{O}_{2}: \mathrm{N}_{2}: \mathrm{Ar}=1: 1: 1\right)$ was easily separated at different temperatures through NW-2.97 (see Fig. 6a). $\mathrm{O}_{2}: \mathrm{N}_{2}$ selectivity does not change much with temperature, indicating that both molecules can easily cross the membrane. However, $\mathrm{O}_{2}$ :Ar selectivity is very sensitive to temperature, reaching extremely high selectivities at cryogenic temperatures. Modeling the framework as rigid (blue triangles in Fig. 6) decreases permeation rate due to the constraint in the nanowindow vibrations but slightly raises selectivity.

A narrower nanowindow (see NW-2.73 in Fig. 6b) is much more attractive for separation purposes. Even though permeation rates are lower compared to $\mathrm{NW}-2.97$, its selectivity to $\mathrm{O}_{2}$ is higher and it dramatically increases after lowering the
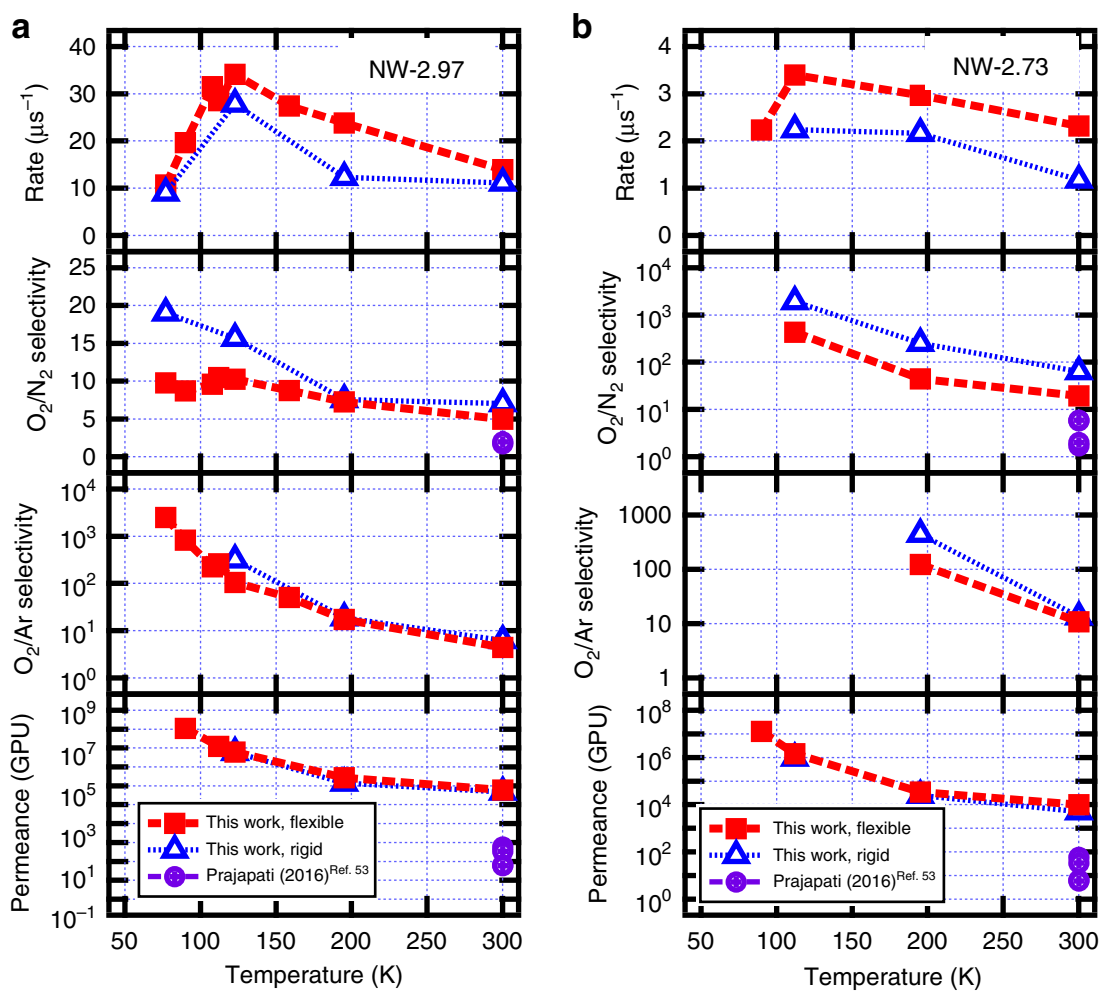

Fig. 6 Nanowindows NW-2.97 and NW-2.73 effectively separate air. Temperature dependence for the various parameters in a NW-2.97 and $\mathbf{b}$ NW-2.73. Optimum rates occur at a temperature with both high kinetic energy and high adsorption amounts. For calculation of GPU $\left(10^{-6} \mathrm{~cm}^{3}(\mathrm{STP}) \mathrm{cm}^{-2} \mathrm{~s}^{-1} \mathrm{~cm}^{-}\right.$ $\mathrm{Hg}^{-1}$ ), the ideal gas pressure was estimated from the density of the gas far from the surface where it behaves as bulk 
temperature. The monotonic decrease of selectivity with rising temperature is a consequence of it being the quotient of the rates of two activated processes. Similarly, the increase of permeance with rising temperature is due to the large changes in gas pressure (the simulation ensemble was canonical) with temperature: in these simulations or air permeation, the calculated pressure was $0.05,0.7,1.3,25$, and 67 bar at $T=90,112,122,195$, and $300 \mathrm{~K}$, respectively. Above $155 \mathrm{~K}$, all these gases are already supercritical.

A more thorough comparison with experimental membranes (see Table 1) shows some crucial points. First, increase in selectivity comes at the cost of decreased permeability. Second, commercial polymers, such as polysulfone, polycarbonate, and polyimides, can only reach permeation rate selectivities of about 6 for $\mathrm{O}_{2}: \mathrm{N}_{2}{ }^{45,46}$. And third, even the best polymer membranes, including those with a mixed matrix, rarely surpass an $\mathrm{O}_{2}: \mathrm{N}_{2}$ selectivity above $10^{45,47-53}$. This can only be obtained at permeability rates many orders of magnitude lower than our graphene nanowindows. This breakthrough in separation membranes becomes clear in a Robeson plot (see Supplementary Fig. 5) where the upper bound is extended by two orders of magnitude if we use our nanowindows at low temperature. This immense potential of ultra-fast separation with graphene nanowindows can be exploited in multistage setups at low temperature, where purity can be quickly raised after each stage.

\section{Discussion}

The behavior of graphene nanowindows of atomic sizes is highly dynamic and dependent on its rim functionalization. Nanowindow breathing, relaxation, rotation of bonds, and large electrostatic fields enable nanowindows to open or close to molecular permeation. Simplified nanowindow models, which ignore the distribution of charges or flexibility of the framework, cannot describe these mechanisms. We showed that high separation selectivities, and especially ultra-fast permeation rates, a thousand times larger than current membranes is achieved and thus encourage further development in description and development of atomically thin membranes for molecular separation.

\footnotetext{
Methods

Nanowindow construction. Graphene nanowindows were modeled by removing carbon atoms from the center of a pristine graphene layer and passivating the exposed edges with $-\mathrm{H},-\mathrm{OH}$, or $\mathrm{C}-\mathrm{O}-\mathrm{C}$ terminations. The nanowindow structure was optimized with MOPAC2016 $6^{54}$ and the PM7 $7^{55}$ method to report the size of the nanowindow as the radius of the largest sphere that fits in the nanowindow, when it just contacts the van der Waals radii of the rim edge atoms.
}

Molecular dynamics. MD simulations were run using $\mathrm{HOOMD}^{56-58}$ code, version 1.3.3. The whole graphene nanowindow framework was considered fully flexible with partial charges along the nanowindow rim. Ar was modeled as a single center LJ particle, whereas $\mathrm{O}_{2}$ and $\mathrm{N}_{2}$ were modeled as rigid bodies consisting of two LJ particles plus three point charges, to take into account their quadrupole moments. A comparison of the charges considered in our models against those calculated by density functional theory (DFT) is shown in Supplementary Fig. 6. Bonded and non-bonded force field parameters are listed in Supplementary Tables 2 and 3. Vibrational frequencies obtained for a hydroxyl-coronene molecule with this force field are compared against those of a high-level DFT calculation in Supplementary Fig. 7. The vibrations playing a key role in permeability, such as $-\mathrm{H}$ switching sides of the graphene layer, have an error of $<3.5 \%$ (see Supplementary Table 4). All crossed LJ interactions were calculated with Lorentz-Berthelot rules. Long-range electrostatics employed an implementation of the particle-particle-

particle-mesh (PPPM) method ${ }^{59}$. The timestep employed in simulations was 1.1 or $8.9 \mathrm{fs}$ for flexible and rigid nanowindows, respectively. Dimensions of the simulation box in the $x, y$, and $z$ directions were $42.63 \AA, 39.38 \AA$, and $50.00 \AA$, respectively. The graphene layer was placed in the $x-y$ plane. Periodic boundary conditions were employed in $x$ and $y$ directions. A reflective $\mathrm{LJ}$ wall was placed at $z$ $=25 \AA$ from the graphene plane.

The graphene framework and nanowindow functional groups were kept flexible in most simulations, except in those with rigid nanowindows and the section of nanowindow dihedral rotation. In the latter, the system was equilibrated with a fully flexible framework at the system temperature. Later, the framework was quenched rigid and its energy minimized followed by another equilibration run and collection.

All molecular models in the figures were prepared using VMD 1.9.360 and rendered with Tachyon ${ }^{61}$.

Data availability. The data that support the findings of this study are available from the corresponding author F. V.-B. on reasonable request.

Received: 23 June 2017 Accepted: 14 April 2018

Published online: 04 May 2018

\section{References}

1. Humphrey, J. L. \& Keller, G. E. Separation Process Technology (McGraw-Hill, 1997).

2. Sholl, D. S. \& Lively, R. P. Seven chemical separations to change the world. Nature 532, 435-437 (2016).

3. Gadalla, M. A., Olujic, Z., Jansens, P. J., Jobson, M. \& Smith, R. Reducing $\mathrm{CO}_{2}$ emissions and energy consumption of heat-integrated distillation systems. Environ. Sci. Technol. 39, 6860-6870 (2005).

4. Gutiérrez-Guerra, R., Segovia-Hernández, J. G. \& Hernández, S. Reducing energy consumption and $\mathrm{CO}_{2}$ emissions in extractive distillation. Chem. Eng. Res. Des. 87, 145-152 (2009).

5. Freemantle, M. Membranes for gas separation: advanced organic and inorganic materials being developed for separations offer cost benefits for environmental and energy-related processes. Chem. Eng. News 83, 49-57 (2005).

6. Huang, L., Zhang, M., Li, C. \& Shi, G. Graphene-based membranes for molecular separation. J. Phys. Chem. Lett. 6, 2806-2815 (2015).

7. Liu, G., Jin, W. \& Xu, N. Graphene-based membranes. Chem. Soc. Rev. 44, 5016-5030 (2015)

8. $\mathrm{Xu}, \mathrm{Q}$. et al. Graphene and graphene oxide: advanced membranes for gas separation and water purification. Inorg. Chem. Front. 2, 417-424 (2015).

9. Werber, J. R., Osuji, C. O. \& Elimelech, M. Materials for next-generation desalination and water purification membranes. Nat. Rev. Mater. 1, 16018 (2016).

10. Kerry, F. G. Industrial Gas Handbook: Gas Separation and Purification (Taylor \& Francis, 2007).

11. Bunch, J. S. et al. Impermeable atomic membranes from graphene sheets. Nano. Lett. 8, 2458-2462 (2008).

12. Surwade, S. P. et al. Water desalination using nanoporous single-layer graphene. Nat. Nanotechnol. 10, 459-464 (2015).

13. Murata, K. et al. Nanowindow-induced molecular sieving effect in a singlewall carbon nanohorn. J. Phys. Chem. B. 106, 12668-12669 (2002).

14. Yang, C.-M. et al. Nanowindow-regulated specific capacitance of supercapacitor electrodes of single-wall carbon nanohorns. J. Am. Chem. Soc. 129, 20-21 (2007).

15. Lehtinen, O. et al. Effects of ion bombardment on a two-dimensional target: Atomistic simulations of graphene irradiation. Phys. Rev. B 81, 153401 (2010).

16. Bieri, M. et al. Porous graphenes: two-dimensional polymer synthesis with atomic precision. Chem. Commun. 6919-6921 (2009).

17. Murata, K. et al. Porosity evaluation of intrinsic intraparticle nanopores of single wall carbon nanohorn. Nano. Lett. 1, 197-199 (2001).

18. Du, H. et al. Separation of hydrogen and nitrogen gases with porous graphene membrane. J. Phys. Chem. C. 115, 23261-23266 (2011).

19. Sun, C. et al. Mechanisms of molecular permeation through nanoporous graphene membranes. Langmuir 30, 675-682 (2014).

20. Azamat, J., Khataee, A. \& Joo, S. W. Molecular dynamics simulation of trihalomethanes separation from water by functionalized nanoporous graphene under induced pressure. Chem. Eng. Sci. 127, 285-292 (2015).

21. Ohba, T. Consecutive water transport through zero-dimensional graphene gates of single-walled carbon nanohorns. J. Phys. Chem. C. 120, 8855-8862 (2016).

22. Tao, Y. et al. Tunable hydrogen separation in porous graphene membrane: first-principle and molecular dynamic simulation. ACS Appl. Mater. Interfaces 6, 8048-8058 (2014).

23. Raghavan, B. \& Gupta, T. $\mathrm{H}_{2} / \mathrm{CH}_{4}$ gas separation by variation in pore geometry of nanoporous graphene. J. Phys. Chem. C 121, 1904-1909 (2017).

24. Hauser, A. W. \& Schwerdtfeger, P. Methane-selective nanoporous graphene membranes for gas purification. Phys. Chem. Chem. Phys. 14, 13292-13298 (2012).

25. Dubbeldam, D., Walton, K. S., Ellis, D. E. \& Snurr, R. Q. Exceptional negative thermal expansion in isoreticular metal-organic frameworks. Angew. Chem. Int. Ed. 46, 4496-4499 (2007). 
26. Keskin, S., Liu, J., Rankin, R. B., Johnson, J. K. \& Sholl, D. S. Progress, opportunities, and challenges for applying atomically detailed modeling to molecular adsorption and transport in metal-organic framework materials. Ind. Eng. Chem. Res. 48, 2355-2371 (2009).

27. Goj, A., Sholl, D. S., Akten, E. D. \& Kohen, D. Atomistic simulations of $\mathrm{CO}_{2}$ and $\mathrm{N}_{2}$ adsorption in silica zeolites: the impact of pore size and shape. J. Phys. Chem. B 106, 8367-8375 (2002).

28. He, Z., Zhou, J., Lu, X. \& Corry, B. Bioinspired graphene nanopores with voltage-tunable ion selectivity for $\mathrm{Na}^{+}$and $\mathrm{K}^{+}$. ACS Nano 7, 10148-10157 (2013).

29. Wen, B., Sun, C. \& Bai, B. Inhibition effect of a non-permeating component on gas permeability of nanoporous graphene membranes. Phys. Chem. Chem. Phys. 17, 23619-23626 (2015).

30. Sun, C., Wen, B. \& Bai, B. Application of nanoporous graphene membranes in natural gas processing: molecular simulations of $\mathrm{CH}_{4} / \mathrm{CO}_{2}, \mathrm{CH}_{4} / \mathrm{H}_{2} \mathrm{~S}$ and $\mathrm{CH}_{4} / \mathrm{N}_{2}$ separation. Chem. Eng. Sci. 138, 616-621 (2015).

31. Sing, K. S. W. \& Williams, R. T. The use of molecular probes for the characterization of nanoporous adsorbents. Part. Part. Syst. Charact. 21, 71-79 (2004).

32. Utsumi, S. et al. Direct evidence on $\mathrm{C}-\mathrm{C}$ single bonding in single-wall carbon nanohorn aggregates. J. Phys. Chem. C. 111, 5572-5575 (2007)

33. Berman, D., Deshmukh, S. A., Sankaranarayanan, S. K. R. S., Erdemir, A. \& Sumant, A. V. Extraordinary macroscale wear resistance of one atom thick graphene layer. Adv. Funct. Mater. 24, 6640-6646 (2014).

34. Liu, Z. et al. Controlling and formation mechanism of oxygen-containing groups on graphite oxide. Ind. Eng. Chem. Res. 53, 253-258 (2014).

35. Dreyer, D. R., Park, S., Bielawski, C. W. \& Ruoff, R. S. The chemistry of graphene oxide. Chem. Soc. Rev. 39, 228-240 (2009).

36. Tararan, A., Zobelli, A., Benito, A. M., Maser, W. K. \& Stéphan, O. Revisiting graphene oxide chemistry via spatially-resolved electron energy loss spectroscopy. Chem. Mater. 28, 3741-3748 (2016).

37. Bagri, A. et al. Structural evolution during the reduction of chemically derived graphene oxide. Nat. Chem. 2, 581-587 (2010).

38. Guo, J. et al. Crown ethers in graphene. Nat. Commun. 5, 5389 (2014).

39. Yamada, Y. et al. Subnanometer vacancy defects introduced on graphene by oxygen gas. J. Am. Chem. Soc. 136, 2232-2235 (2014).

40. Drahushuk, L. W. \& Strano, M. S. Mechanisms of gas permeation through single layer graphene membranes. Langmuir 28, 16671-16678 (2012).

41. Drahushuk, L. W., Wang, L., Koenig, S. P., Bunch, J. S. \& Strano, M. S Analysis of time-varying, stochastic gas transport through graphene membranes. ACS Nano 10, 786-795 (2016).

42. Yuan, Z. et al. Mechanism and prediction of gas permeation through subnanometer graphene pores: comparison of theory and simulation. ACS Nano 11, 7974-7987 (2017).

43. Zhang, K. et al. Exploring the framework hydrophobicity and flexibility of ZIF-8: from biofuel recovery to hydrocarbon separations. J. Phys. Chem. Lett. 4, 3618-3622 (2013).

44. Leroy, F., Rousseau, B. \& Fuchs, A. H. Self-diffusion of n-alkanes in silicalite using molecular dynamics simulation: a comparison between rigid and flexible frameworks. Phys. Chem. Chem. Phys. 6, 775-783 (2004).

45. Murali, R. S., Sankarshana, T. \& Sridhar, S. Air separation by polymer-based membrane technology. Sep. Purif. Rev. 42, 130-186 (2013).

46. Thorogood, R. M. Air separation. Access Sci. https://doi.org/10.1036/10978542.059400 (2014).

47. Kesting, R. E., Fritzsche, A. K., Cruse, C. A. \& Moore, M. D. The secondgeneration polysulfone gas-separation membrane. II. The relationship between sol properties, gel microvoids, and fiber selectivity. J. Appl. Polym. Sci. 40, 1575-1582 (1990).

48. Pesek, S. C. \& Koros, W. J. Aqueous quenched asymmetric polysulfone hollow fibers prepared by dry/wet phase separation. J. Membr. Sci. 88, 1-19 (1994).

49. Chung, T.-S., Lin, W.-H. \& Vora, R. H. The effect of shear rates on gas separation performance of 6FDA-durene polyimide hollow fibers. J. Membr. Sci. 167, 55-66 (2000).

50. Clausi, D. T. \& Koros, W. J. Formation of defect-free polyimide hollow fiber membranes for gas separations. J. Membr. Sci. 167, 79-89 (2000).

51. Sterescu, D. M. et al. Boltorn-modified poly(2,6-dimethyl-1,4-phenylene oxide) gas separation membranes. Macromolecules 40, 5400-5410 (2007).
52. Liu, C., Minkov, R., Faheem, S. A., Bowen, T. C. \& Chiou, J. J. High permeance polyimide membranes for air separation. Patent EP 2576032 A4 (2013).

53. Prajapati, P. K., Kansara, A. M. \& Singh, P. S. Preparation and characterization of an oxygen permselective polydimethylsiloxane hollow fibre membrane. RSC Adv. 6, 88943-88953 (2016).

54. Stewart, J. J. P. MOPAC 2016 (Stewart Computational Chemistry) (2016).

55. Stewart, J. J. P. Optimization of parameters for semiempirical methods VI: more modifications to the NDDO approximations and re-optimization of parameters. J. Mol. Model. 19, 1-32 (2013).

56. Anderson, J. A., Lorenz, C. D. \& Travesset, A. General purpose molecular dynamics simulations fully implemented on graphics processing units. J. Comput. Phys. 227, 5342-5359 (2008).

57. Glaser, J. et al. Strong scaling of general-purpose molecular dynamics simulations on GPUs. Comput. Phys. Commun. 192, 97-107 (2015).

58. Nguyen, T. D., Phillips, C. L., Anderson, J. A. \& Glotzer, S. C. Rigid body constraints realized in massively-parallel molecular dynamics on graphics processing units. Comput. Phys. Commun. 182, 2307-2313 (2011).

59. LeBard, D. N. et al. Self-assembly of coarse-grained ionic surfactants accelerated by graphics processing units. Soft Matter 8, 2385-2397 (2012).

60. Humphrey, W., Dalke, A. \& Schulten, K. VMD: visual molecular dynamics. J. Mol. Graph. 14, 33-38 (1996).

61. Stone, J. An efficient library for parallel ray tracing and animation (Computer Science Department, University of Missouri-Rolla, 1998).

\section{Acknowledgements}

F.V-B. is supported by TAKAGI Co., Ltd. F.V-B. and K.K. acknowledge project JST CREST "Creation of Innovative Functional Materials with Advanced Properties by Hyper-nanospace Design”. This work was partially supported by the grant of OPERA project from JST.

\section{Author contributions}

F.V-B. performed all molecular simulations and prepared the figures. K.K. and F.V-B. conceived the idea. All authors analyzed the data, commented the results, and wrote the manuscript.

\section{Additional information}

Supplementary Information accompanies this paper at https://doi.org/10.1038/s41467018-04224-6.

Competing interests: The authors declare no competing interests.

Reprints and permission information is available online at http://npg.nature.com/ reprintsandpermissions/

Publisher's note: Springer Nature remains neutral with regard to jurisdictional claims in published maps and institutional affiliations.

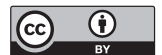

Open Access This article is licensed under a Creative Commons Attribution 4.0 International License, which permits use, sharing, adaptation, distribution and reproduction in any medium or format, as long as you give appropriate credit to the original author(s) and the source, provide a link to the Creative Commons license, and indicate if changes were made. The images or other third party material in this article are included in the article's Creative Commons license, unless indicated otherwise in a credit line to the material. If material is not included in the article's Creative Commons license and your intended use is not permitted by statutory regulation or exceeds the permitted use, you will need to obtain permission directly from the copyright holder. To view a copy of this license, visit http://creativecommons.org/ licenses/by/4.0/.

(C) The Author(s) 2018 